\title{
The mispriming effect: Evidence of an orthographic check in the lexical decision task
}

\author{
D. NORRIS \\ Medical Research Council, Applied Psychology Unit, Cambridge, England
}

\begin{abstract}
This paper describes a lexical decision experiment that demonstrates that there are increased rates of errors for words "misprimed" with words strongly associated with visually similar words (e.g., BREAD-BATTER) relative to rates for the same words preceded by completely unrelated words (e.g., SLEEP-BATTER). The pattern of results is shown to be inconsistent with Forster's (1976) search model but consistent with a criterion-bias model supplemented by an orthographic checking process.
\end{abstract}

We know that in the case of nonwords that are visually very similar to words, the presence of some contextual evidence in favor of the visually similar words can override the perceptual evidence in favor of the nonwords and lead to an increase in error rate (O'Connor \& Forster, 1981). But what happens in the case of two very similar words (e.g., BATTER, BUTTER) when we present subjects with one of these words preceded by a word strongly related to the other word (e.g., BREAD-BATTER)?

When words are "misprimed" in this way, simple criterion-bias accounts of context effects and search models make very different predictions about performance in the lexical decision task. The most straightforward predictions follow from a criterion-bias account of context effects. The effect of a prime (BREAD) should be to reduce the recognition criterion for related words (e.g., BUTTER). If the criterion reduction for BUTTER is sufficiently large, then the visually similar word BATTER will provide enough perceptual information in support of BUTTER for the subject to identify the stimulus as BUTTER. The misprimed stimulus should therefore be correctly classified as a word, but for the wrong reason. When BATTER is not misprimed, the criterion for BUTTER will not be reduced and the probability of misidentifying BATTER as BUTTER should be greatly decreased. A criterion-bias model should therefore predict that if there is any difference in error rate between misprimed and unprimed trials, it should be in the direction of fewer errors on misprimed trials, since these words will be correctly classified as words if there is sufficient evidence for them to be identified as either the word presented or its visually similar primed counterpart.

A criterion-bias model should also predict that if there is any difference in speed of classification of misprimed

The research reported in the paper was supported by Grant HR 7147 from the Social Science Research Council of Great Britain. The theoretical interpretation of the data presented in the paper evolved during the course of conversation with Jim Chumbley. The author's mailing address is: Medical Research Council, Applied Psychology Unit, 15 Chaucer Road, Cambridge CB2 2EF, England. and unrelated words, the misprimed words will be classified faster than the unrelated words. When BATTER is misprimed, it will be correctly classified as soon as either the threshold for BATTER or the reduced threshold for BUTTER is exceeded. However, an unrelated word can be classified only whenever its own threshold is exceeded.

Forster's (1976) search model makes a rather different set of predictions. According to O'Connor and Forster's (1981) interpretation of the model, we should expect misprimed words to be misclassified as nonwords on some trials. O'Connor and Forster argued that "No" responses in a lexical decision task are sometimes executed when the target string is found to differ only slightly from a lexical entry encountered in the search. In the search model, words can be identified on the basis of either a frequencyordered or a semantically driven search. This results in two ways in which a relatively low-frequency word such as BATTER could be erroneously classified as a nonword. If either the frequency or the semantic search encounters a visually similar word, such as BUTTER, before they encounter BATTER, then there will be an opportunity to classify BATTER as a nonword. Therefore, even in the absence of context, a low-frequency word will have a relatively high error rate because it will sometimes be classified as a nonword when the frequency-ordered search encounters a visually similar word of higher frequency. However, if BATTER is preceded by the word BREAD, BUTTER will now be encountered in both the frequency search and the semantic search. There will therefore be two opportunities for BATTER to be misclassified as a nonword. Priming will therefore increase the probability that BATTER will be incorrectly classified as a nonword. However, on trials when BATTER is classified correctly, the classification will be made on the basis of the frequency-ordered search, and there should be no difference in the time taken to classify misprimed and unprimed words.

The simple criterion-bias model and the search model therefore make almost completely opposite predictions about both speed and accuracy of classifying misprimed words in a lexical decision task. Criterion-bias models 
predict that misprimed trials should tend to be classified faster and more accurately than unprimed trials, whereas Forster's (1976) search model predicts a greater incidence of errors in the classification of misprimed trials, but predicts no difference in classification latency. The present experiment was designed to distinguish between these two theories.

\section{METHOD}

In order to assess whether any observable differences between misprimed and unprimed trials could be due to the operation of strategies specific to a single experimental situation, the task demands of the experiment were varied by altering the nature of the nonword trials.

The alterations in the nonword trials produced three different experimental contexts. In the first of the experimental contexts, half of the nonword trials were created by altering a single letter in the second member of a highly associated word pair, such as AUNT-UNCLE, to produce a word-nonword pair, such as AUNT-UMCLE. This condition was designed to maximize the probability of misprimed words' being classified as nonwords, by inducing subjects to adopt a strategy of classifying strings that differed slightly from primed words as nonwords. Also, in terms of criterion-bias models, this condition should tend to reduce the value of the priming information, because if the recognition criterion is reduced too far, UMCLE will be misclassified as UNCLE.

In the second context condition, the words and nonwords from AUNTUMCLE pairs were reassigned, such that none of the nonwords was ever visually similar to a primed word. Subjects in this condition should, therefore, not be misled by the priming information, and the overall value of the primes should increase. Also, there will no longer be any motivation for subjects to adopt a strategy of classifying as nonwords strings that are similar to primed words, since the only effect of this strategy will be to lead to errors on misprimed word trials.

In the final context condition, half of the nonwords were replaced with nonwords that were orthographically regular but that differed from any real word by more than a single letter. This manipulation should make the word-nonword discrimination easier; in criterion-bias terms, it should lead to an overall reduction in the criterion for responding "Yes." With a low criterion, the probability of misidentifying the misprimed word as the primed word should increase, and therefore this condition should have the greatest probability of producing faster classification latencies to misprimed than to unprimed trials.

In order to minimize the possibility that the subjects might consciously examine each word letter by letter, the experimental instructions placed a strong emphasis on speed rather than on accuracy.

\section{Materials}

The experimental materials were constructed around two sets of 11 misprimed word pairs. Unrelated word pairs were created by altering the pairing of primes and targets in such a way as to eliminate any obvious semantic or associative relationship. In one half of the experimental lists, one set of words appeared in the misprimed condition and the other in the unrelated condition, and in the remaining lists, this assignment to conditions was reversed. In addition to the experimental trials, each list contained 78 filler trials: 30 related word pairs, 8 unrelated word pairs, and 40 word-nonword pairs. The filler word trials were predominantly related, so that with experimental trials classified as "unrelated," there were equal numbers of related and unrelated trials. In Conditions 1 and 2, all nonwords were created by altering a single letter in the second member of a highly associated word pair (e.g., DEADALAVE, AUNT-UMCLE). In Condition 1, the words and nonwords in half of the pairs were rearranged to eliminate all associative relationships between the word from which the nonword was derived and the preceding prime. In Condition 2, this rearrangement was performed on all word-nonword pairs so that no nonword was derived from a word related to its prime.

Condition 3 was similar to Condition 2, but half of the nonwords were replaced by nonwords that differed from any real word by at least two letters. These new nonwords replaced items that either were pseudohomophones $(n=7)$ or gave the appearance of being misspellings of real words (e.g., LEDTER, UMCLE).

The experimental words and nonwords are listed in Appendixes $A$ and B. The mean Kučera and Francis (1968) frequency of the experimiental words was 36, and that of their visually similar counterparts was 293 . Filler words had a mean frequency of 173 . For each condition, a practice list of 18 trials was constructed similarly to the corresponding experimental list, but with no misprimed trials.

\section{Subjects}

The subjects were 39 students from the University of Sussex, who were each paid 80 pence.

\section{Procedure}

Stimuli were presented in uppercase letters on a TeleVideo 912 terminal with a white phosphor. Stimulus presentation and response timing were controlled by a Motorola 6809 microprocessor. Each prime word was displayed for $0.5 \mathrm{sec}$ and was followed immediately by the letter string, which was presented for $2.5 \mathrm{sec}$ or until the subject responded. There was a $1.5-\mathrm{sec}$ interval between trials.

The subjects were instructed to respond as quickly as possible. They were told to press a button labeled "Yes" on a response box in front of them with their dominant hand if the stimulus was a word and one labeled "No" with their nondominant hand if it was a nonsense word. The experimenter emphasized that speed was far more important than accuracy and that it would be quite acceptable to make as many as $25 \%-30 \%$ errors in the interest of responding quickly. Because the primary interest in this experiment was the relative error rates to misprimed and unprimed experimental trials, subjects who made no errors at all on experimental trials were replaced. The subjects were assigned to conditions in rotation as they appeared for the experiment.

\section{RESULTS}

The data from 3 subjects, 1 from Condition 2 and 2 from Condition 3, were discarded because those subjects made no errors on any of the experimental trials. All analyses are based on the data from 36 subjects, 12 in each condition.

The results are shown in Table 1.

Note that no materials analyses are presented in this paper. However, data for individual items are presented in Appendix A. All of the conclusions drawn from the data

Table 1

Mean Error Rates (in Percent) and Lexical Decision Latencies (RTs, in Milliseconds) to Words

\begin{tabular}{|c|c|c|c|c|c|c|c|c|c|}
\hline \multirow[b]{2}{*}{ Condition } & \multicolumn{3}{|c|}{ Misprimed (BREAD-BATTER) } & \multicolumn{3}{|c|}{ Unrelated (UP-BATTER) } & \multicolumn{3}{|c|}{ Mean } \\
\hline & Error Rate & Error RT & Correct RT & Error Rate & Error RT & Correct RT & Error Rate & Error RT & Correct RT \\
\hline 1 & 32 & 663 & 680 & 22 & 653 & 644 & 27 & 658 & 662 \\
\hline 2 & 27 & 681 & 658 & 19 & 689 & 631 & 23 & 685 & 644 \\
\hline 3 & 24 & 733 & 621 & 11 & 728 & 656 & 17 & 730 & 638 \\
\hline Mean & 28 & 696 & 653 & 17 & 690 & 643 & & & \\
\hline
\end{tabular}


rely simply on the fact that a single set of materials exists that behaves in a manner that is incompatible with some theories but consistent with others. In principle, the main null hypotheses being tested could be rejected even if only a single word were found that demonstrated a significant effect when none was predicted by the null hypothesis. For example, a single word that gave rise to a reliably higher error rate in the misprimed than in the unrelated condition would be sufficient to reject a simple criterion-bias model. Such a model predicts that error rates for all words should be greatest in the unrelated condition. ${ }^{1}$

Analysis of the error data showed that the effects of both experimental context $[\mathrm{F}(2,30)=4.211, \mathrm{p}<0.025]$ and priming $[F(1,30)=16.095, p<.0005]$ were significant, but neither the interaction of context and mispriming ( $F$ $<1)$ nor any other effects were significant.

A further analysis of variance was performed on latencies to all experimental trials with correctness of response ("Yes" vs. "No") as an additional factor. This analysis revealed a highly significant effect of correctness of response $[F(1,30)=17.012, p<.0005]$, with "Yes" responses being faster than "No" responses, and an interaction between correctness and experimental context $[\mathrm{F}(2,30=6.115, \mathrm{p}<.01]$, with the context condition producing the fastest "Yes" responses (Condition 3 ) also producing the slowest "No" responses. No other effects were significant.

The results for the filler word trials are shown in Table 2. The main effect of priming was significant in both the latency $[\mathrm{F}(1,30)=35.57, \mathrm{p}<.0005]$ and in the error $[F(1,30)=74.91, p<.0005]$ analyses. No other effects were significant.

\section{DISCUSSION}

The only aspect of the data compatible with a simple criterion-bias model is the slight tendency for misprimed words to be correctly classified faster than unprimed words in Condition 3. However, in Condition 1, this effect actually goes in the opposite direction, with misprimed words being slightly harder to classify.

Superficially, these results appear to provide strong support for the search model; as this model predicts, there is a higher error rate for misprimed than for unprimed words. It is interesting to note, however, that this difference in error rates remains fairly constant across the three context conditions. Most significantly, there is no evidence that the tendency to misclassify misprimed words is greatest in Condition 1, in which the subjects might have

Table 2

Mean Error Rates (in Percent) and Lexical Decision Latencies (RTs, in Milliseconds) to Filler Words

\begin{tabular}{cccccc}
\hline & \multicolumn{2}{c}{ Primed } & & \multicolumn{2}{c}{ Unrelated } \\
\cline { 2 - 3 } \cline { 5 - 6 } Condition & Error Rate & Correct RT & & Error Rate & Correct RT \\
\hline 1 & 3.3 & 547 & & 17.7 & 585 \\
2 & 2.5 & 552 & & 19.8 & 583 \\
3 & 1.4 & 523 & & 13.5 & 579 \\
\hline
\end{tabular}

been expected to adopt a strategy of classifying strings that differed only slightly from primed words as nonwords.

Although the error data are consistent with the search model, however, the latency data are not. According to Forster's (1976) search model, errors on unprimed trials should be due to subjects' misclassifying BATTER when it is found to be only slightly different from BUTTER during the frequency-ordered search. Some of the errors to misprimed trials will also be due to the frequency-ordered search. However, any errors on misprimed trials beyond the number of errors on unprimed trials should be due to misclassification during the semantically driven search. Furthermore, errors attributable to the semantic search should be faster than errors attributable to the frequency ordered search. This is because the semantically driven search should reach the entry for BUTTER faster than the frequency-ordered search. (The assumption that the semantic search is faster than the frequency search is the central assumption underlying the priming effect in the search model.) This means that the greater the proportion of errors due to the semantic search, the faster will be the mean error response. The proportion of errors in the misprimed condition due to the semantic search is given by the difference between the number of errors in misprimed and unrelated conditions divided by the total number of errors in these conditions. Therefore, the fastest errors should be made in Condition 3 , in which the proportion of semantic errors is greatest $[(24.2-10.6) /(24.2+$ $10.6)=0.39$ ] and the "Yes" responses are fastest; the slowest errors should be in Condition 1, in which the proportion of semantic errors is smallest [(31.8 $22) /(31.8+22)=0.18$ ] and the "Yes" responses are the slowest. However, the fastest errors are actually made in Condition 1, in which the correct response latencies are slowest and there is the smallest proportion of semantic errors.

Although this interaction of correctness and context is very difficult to accommodate within the framework of a search model, a simple addition to a criterion-bias model can provide an accurate account of both the latency and the error data.

A criticism frequently directed at criterion-bias models, particularly those based on the logogen model (Morton, 1969), is that they contain no mechanism for making detailed discriminations between words and nonwords. Coltheart, Davelaar, Jonasson, and Besner (1977) suggested that "No" responses in a lexical decision task can be executed once a certain period of time during which there is no further activation of logogens has elapsed. However, such an account cannot explain the results of the present experiment, in which it was the similarity between the presented word and a word related to the context that led to errors. The present results strongly suggest that, on certain trials at least, subjects are performing some kind of spelling check on the stimulus. The effect of mispriming is to cause the primed word (BUTTER), rather than the presented word (BATTER), to be used as the reference for the check. When this occurs, the check will fail-BUTTER is not spelled B,A,T,T,E,R-and the 
stimulus will be classified as a nonword. Any proposals that a check is carried out on all trials can be dismissed very rapidly, since such a model would also run into problems in explaining the interaction between context condition and correctness in the latency data. If all errors were due to the failure of a spelling check, then error response latencies should be determined by the time taken to generate a candidate analysis for the check and the time taken to generate a candidate should be positively correlated with the time taken to correctly recognize a word.

The need for an orthographic check will be greatest when there is inadequate information to make either a "Yes" or a "No" response with confidence. If the evidence in favor of a word is very strong, then the check would be unnecessary. If there is no sound evidence in favor of any word, on the other hand, a "No" response could be made with confidence. It is with intermediate levels of evidence that a spelling check would resolve the uncertainty and enable a response to be made.

In effect, what is being proposed is a synthesis of the Coltheart et al. (1977) response-deadline model with a two-stage decision model similar to that of Atkinson and Juola (1973) or to that proposed by Rips, Shoben, and Smith (1973) for the semantic verification task. In contrast with the single response criterion proposed by Coltheart et al., this model assumes that subjects have both an upper and a lower response criterion. Only if the evidence accumulated with the response deadline exceeds the upper criterion can a fast "Yes" response be made. A fast "No" response will be made only of the evidence for all words fails to reach the lower criterion within the deadline. If the deadline expires and the evidence for one or more words falls between the two criteria, then the system is assumed to carry out a check on the spelling of the stimulus by using the word for which there is most evidence as a reference. This spelling check is assumed to be a relatively time-consuming process. If the check succeeds and the stimulus is found to be spelled in the same way as the reference word, the subject will respond "Yes." If the stimulus is spelled differently from the reference word, the check will fail and the subject will respond "No." It is assumed that the positioning of the two criteria can be adjusted independently to optimize performance under different conditions.

In order to account for the present data, it is assumed that mispriming frequently results in there being more evidence for the visually similar primed word (BUTTER) than for the word actually presented (BATTER). If the evidence for BUTTER falls in the region between the two criteria and is greater than the evidence for BATTER, then BUTTER will be used as the reference for the check. Under these circumstances, the check will fail and the subject will therefore wrongly classify BATTER as a nonword. Thus, the mispriming effect is due to the fact that, by priming a word that is visually similar to the stimulus, the system will occasionally be led to select the wrong word as the reference for the spelling check. In order to provide an account of the differences between the context conditions, the model also has to assume that subjects adopt a higher upper criterion for the relatively difficult discriminations required in Conditions 1 and 2 than they do for Condition 3.

Thus, in Condition 1, some of the experimental words will not reach the relatively high lower criterion and will therefore be rejected rapidly as nonwords. Words that do not exceed the lower criterion will be classified as nonwords regardless of whether the evidence favors the presented word or the primed word. Similarly, any word that exceeds the higher criterion will be quickly classified as a word. If the evidence falls between the two criteria, a spelling check will be performed. If the evidence favors the presented word (BATTER), then the check will succeed and a correct "Yes" response will be made. If the evidence favors the primed word (BUTTER), the check will fail and a slow error response will be made.

This two-stage decision model makes the opposite predictions about error response latencies from the search model. According to the two-stage model, the extra errors to misprimed in comparison with unprimed words are due to the operation of the spelling check using the primed words as references, whereas errors made to both misprimed and unprimed words will be mainly due to fast "No" responses when the evidence does not exceed the lower decision criterion. This predicts that the error response latencies should depend on the proportion of errors due to the spelling check. The more errors due to the spelling check, the slower will be the overall error response latency. The proportion of errors due to each source is calculated by the same procedure as the proportion of frequency and semantic errors in the search model. Therefore, the fastest error responses will be expected in Condition 1, in which the proportion of errors due to the spelling check is smallest, and the slowest will be in Condition 3 , in which the operation of the spelling check will account for over one-third of the errors. This is exactly what was found. (Note here that if all unrelated errors were due to fast "No" responses, then unrelated error response times should actually be faster in Condition 3 than in either Condition 1 or Condition 2. The lower error rate in Condition 3 suggests that the lower criterion has been reduced. In order to account for the increase in error response time actually found in Condition 3, it must be assumed that the spelling check is responsible for a higher proportion of unrelated errors in Condition 3 than in Conditions 1 and 2.)

The two-stage decision model thus provides an accurate account of both error rates and latencies for " $\mathrm{No}^{\circ}$ responses, and it also accounts for the observed pattern of latencies for "Yes" responses. The fast latencies and low error rates to misprimed trials in Condition 3 suggest that subjects are adopting an upper criterion lower than the criteria in the other conditions. With a low upper criterion, there is a greater chance that mispriming will 
result in the primed words' exceeding the upper criterion and being quickly classified as words. (This is the basic prediction derived above from criterion-bias models.) With a higher upper criterion, on the other hand, the probability of misidentifying misprimed words will decrease and "Yes" latencies should no longer demonstrate an advantage due to misidentification.

Overall, the two-stage decision model provides a very accurate account of the way in which the pattern of reaction times and errors changes from Condition 1 to Condition 3. This pattern of data is totally inconsistent with both the logogen model and the search model. However, the two-stage decision model does make one prediction that is not borne out by the data. It predicts that correct responses to misprimed trials should be faster than correct responses to unrelated trials. The logic behind this prediction is similar to that behind the logogen model's prediction that correct responses should be faster to misprimed trials. A correct "Yes"' response can be initiated either when the evidence for a word exceeds the upper criterion, in which case a fast response will be made, or when the correct word is selected as the reference for the spelling check, in which case a slow response will be made. In the case of either an unrelated or a misprimed word, the spelling check can lead to a correct response only if the correct word is selected as the reference. However, a fast "Yes" can be made as soon as any word exceeds the upper criterion; it need not be the correct word. On misprimed trials, a fast "Yes" can be made when either the presented word (BATTER) or the visually similar primed word (BUTTER) exceeds the upper criterion. However, on unrelated trials, there will be only a relatively small probability that the visually similar word will exceed the upper criterion. Therefore, there will be a smaller proportion of fast "Yes" responses to unrelated trials, and correct responses to unrelated trials should be slower than those to misprimed trials. However, correct responses to unrelated trials are actually $10 \mathrm{msec}$ faster than responses to misprimed trials. Although this small difference could be dismissed as insignificant in view of the data for which the model correctly accounts, a fairly simple modification of the model can suggest why responses to misprimed trials should be slower.

Most of the model's predictions derive from the assumption that on misprimed trials the evidence for the primed word is sometimes greater than the evidence for the word actually presented. Sometimes the presented word will be misidentified as the primed word, and sometimes the primed word will be used wrongly as the reference for the spelling check. However, what is the optimum course of action when the evidence for both the primed and the presented words is almost identical, yet falls between the two criteria? When there is little evidence to favor one word over the other, the spelling check is going to fail on about half of the trials on which a word is presented. The real benefit of the spelling check can be obtained only when the system knows that, if the target is a word, it is the word for which there is the most evidence. There are two ways in which the system could be changed to reduce the probability of errors in instances in which there is similar evidence for two or more words. First, the system could perform a check on all words falling between the lower and upper criterion. Alternatively, the system could wait until the evidence begins to favor one word over the other, and then perform a single check on the preferred word. In either case, the net effect would be to lead to slower correct response times. But more importantly, correct responses to misprimed words should be slowed more than responses to unrelated words. Because misprimed trials will be more likely to result in the primed word's (BUTTER) exceeding the lower criterion, misprimed trials will also be more likely to lead to a close tie between the evidence for the primed word and that for the presented word. On unrelated trials, the evidence should usually favor the presented word by a considerable margin. Therefore, if the model is modified to handle ties in either of the ways suggested, it will be able to explain why misprimed trials should sometimes be slower than unrelated trials. Additionally, neither of these modifications should alter the model's predictions about other aspects of the data. Because the modification alters the model's predictions only in cases in which there is very similar evidence for two or more words, the rest of the predictions remain unchanged.

O'Connor and Forster (1981) interpreted their data as providing support for the search model but disconfirming criterion-bias models. They reached this conclusion on the basis that both frequency and context effects are present in a misspelling detection task in which subjects are required to decide whether letter strings are correctly spelled words or are misspellings of words. Using a similar line of reasoning to that of Antos (1979), they argued that "it is difficult to see how the misspelling detection task could be performed without waiting until virtually all orthographic information had been processed" (p. 89). If this task requires all of the orthographic information to be processed, then there can be no reduction in response criterion and there should therefore be no effects of either context or frequency.

However, as long as the task does not actually require that every last drop of orthographic information be extracted from the stimulus, there will still be some room for criterion reduction to take place without a drastic increase in the error rate. The assumption underlying the O'Connor and Forster (1981) argument seems to be that orthographic information must be extracted in letter-sized quanta. Therefore, if the task required every letter to be identified, a quantal reduction in the recognition criterion would be bound to lead to a high error rate. However, if criterion reduction simply involved, say, a $5 \%$ reduction in the number of letter features required, then it is unlikely that this would greatly increase the error rate unless all of the missing features always came from the part of the word that was misspelled. 
O'Connor and Forster (1981) also argued that a criterion-bias model with a postaccess check could not account for their data. However, this argument follows from the rather strange assumptions that the check must operate on exactly the same products of perceptual analysis as those that drive lexical access, and that the stimulus must be checked against a prestored word template. They claimed that such a model would have to predict that there would be no context or frequency effects for words presented in mixed case, whereas their data demonstrate that this is not so. However, the criterion-bias model proposed here has no difficulty in accounting for the existence of context and frequency effects with mixed-case stimuli, since there is no reason that the orthographic check should involve matching against a global word-level template. Context and frequency should influence both lexical access time and the probability of performing an orthographic check. Once lexical access has occurred, an orthographic check can take place at the letter level, rather than at the word level, and will therefore be uninfluenced by case. Presumably, the processes of lexical access and initiating the orthographic check take some time. Therefore, even if the check operates on the output of the same perceptual process as lexical access, that process will have had extra time to analyze the stimulus. The check will therefore have access to more detailed information about the word than was available for lexical access. O'Connor and Forster's criticisms of criterion-bias models therefore apply only to a very restricted class of model. There is nothing in their data that refutes criterion-bias models in general. However, the pattern of results observed in the present experiment is inconsistent with O'Connor and Forster's formulation of the search model.

\section{REFERENCES}

Antos, S. J. (1979). Processing facilitation in a lexical-decision task. Journal of Experimental Psychology, 5, 527-545.

ATKINSON, R. C., \& JUOLA, J. F. (1973). Factors influencing the speed and accuracy of word recognition. In S. Kornblum (Ed.), Attention and performance IV. NY: Academic Press.

Coltheart, M., Davelaar, E., Jonasson, J., \& Besner, D. (1977). Access to the internal lexicon. In S. Dornic (Ed.), Attention and performance VI. Hillsdale, NJ: Erlbaum.

Forster, K. I. (1976). Accessing the mental lexicon. In R. J. Wales \& E. C. T. Walker (Eds.), New approaches to language mechanisms. Amsterdam: North-Holland.

KuČERA, H., \& FrANCIS, W. N. (1967). Computational analysis of presentday American English. Providence, RI: Brown University Press.

MorTon, J. (1969). Interaction of information in word recognition. Psychological Review, 76, 165-178.

O'CONNOR, R. E., \& Forster, K. I. (1981). Criterion bias and search sequence bias in word recognition. Memory \& Cognition, 9, 78-92.

Rirs, L. J., Shoben, E. J., \& SMITH, E. E. (1973). Semantic distance and the verification of semantic relationships. Joumal of Verbal Learning and Verbal Behavior, 12, 1-20.

\section{NOTE}

1. One might wish to generalize from the present results to other pairs of visually similar words in which one of the members of the pairs can be primed. However, it is not clear how one would define the population of visually similar word pairs other than those words differing by a single vowel. But, presumably, what really counts is not the number of letters in common, but the similarity of visual access codes. In fact, there is no way to be sure that all of the word pairs in the present experiment really are visually similar. Perhaps only those that show the mispriming effect are really similar. Without some independent measure of similarity, it is impossible to specify the population of words to which one might wish to generalize, or even the subset of words in the present experiment that are samples from that population. Therefore, none of the statistical analyses presented in this paper attempt to generalize to a new set of materials.

Appendix A

Mean Response Latencies (RTs, in Milliseconds) and Error Rates (in Percent) for Experimental Trials Averaged Across All Three Context Conditions

\begin{tabular}{|c|c|c|c|c|c|c|}
\hline & & & \multicolumn{2}{|c|}{ Related } & \multicolumn{2}{|c|}{ Unrelated } \\
\hline & & & Error Rate & RT & Error Rate & RT \\
\hline BREAD & (BUTTER) & BATTER & 11.1 & 630 & 0.0 & 612 \\
\hline DREAM & (SLEEP) & SLEET & 16.7 & 691 & 44.4 & 798 \\
\hline HARD & (SOFT) & SIFT & 66.7 & 575 & 33.3 & 693 \\
\hline MOON & (STARS) & STIRS & 38.9 & 700 & 0.0 & 644 \\
\hline SHEEP & (LAMB) & LIMB & 33.3 & 553 & 61.1 & 685 \\
\hline SHORT & (TALL) & TELL & 0.0 & 543 & 0.0 & 545 \\
\hline SLEEP & (BED) & BID & 33.3 & 683 & 11.1 & 600 \\
\hline DRY & (WET) & WIT & 44.4 & 681 & 0.0 & 644 \\
\hline PEPPER & (SALT) & SILT & 38.9 & 696 & 33.3 & 762 \\
\hline GREEN & (GRASS) & GROSS & 11.1 & 726 & 11.1 & 644 \\
\hline MEMORY & (MIND) & MEND & 0.0 & 624 & 33.3 & 653 \\
\hline BITTER & (SWEET) & SWEAT & 77.8 & 567 & 0.0 & 585 \\
\hline ALARM & (CLOCK) & CLICK & 5.6 & 681 & 5.6 & 704 \\
\hline YOURS & (MIND) & MANE & 16.7 & 593 & 16.7 & 777 \\
\hline OPEN & (CLOSED) & CLONED & 44.4 & 787 & 38.9 & 782 \\
\hline UP & (DOWN) & DAWN & 50.0 & 867 & 16.7 & 584 \\
\hline HIDE & (SEEK) & SEED & 11.1 & 587 & 16.7 & 623 \\
\hline START & (STOP) & STEP & 16.7 & 681 & 0.0 & 544 \\
\hline FOOT & (HAND) & HIND & 44.4 & 681 & 16.7 & 691 \\
\hline GIRL & (BOY) & BAY & 27.8 & 663 & 11.1 & 582 \\
\hline MUCH & (MORE) & MARE & 22.2 & 642 & 22.2 & 629 \\
\hline NOW & (THEN) & THIN & 0.0 & 553 & 5.6 & 553 \\
\hline
\end{tabular}


Appendix B

Nonword and Filler Items

\begin{tabular}{lllr}
\hline & \multicolumn{2}{c}{ Primed Word-Nonword Pairs for Condition 1 } \\
KITCHEN & SIMK & EAGLE & \\
PURSE & MONAY & TEMPEST & STORN \\
DEAD & ALAVE & CAT & BIRT \\
TALL & SHORD & FOOD & DRONK \\
FISH & CHIBS & RICH & DEG \\
ARM & LIG & LOUD & SAFT \\
NEEDLE & PON & ROUND & POOB \\
JUNE & JILY & SICKNESS & HEALCH \\
TOWN & COUNDRY & ENVELOPE & SQUORE \\
MUTTON & LANB & MONTHS & YEERS \\
HAMMER & NAEL & SCARED & LEDTER \\
FOREST & TREA & LOST & CETS \\
AUNT & UMCLE & MOON & STORS \\
LION & TIDER & GOOD & FOUNB \\
SILVER & GOLB & WINDOW & PAME \\
NEEDLE & THREAB & BOOR \\
FOOD & DRONK & DOOR & SED \\
MAN & WONAM & HAPPY & KNOCKAR \\
SALT & PEPPOR & CARPET & COLB \\
DOCTOR & NIRSE & ICE & ROG \\
& & KITTENS & AFRAED
\end{tabular}

Nonwords used in Condition 3

Differing from word by one letter

$\begin{array}{ll}\text { BIRT } & \text { POLT } \\ \text { KIZE } & \text { POOB } \\ \text { BULAD } & \text { BEFT } \\ \text { ROG } & \text { CHES } \\ \text { KOOD } & \text { DEG } \\ \text { STORN } & \text { CETS } \\ \text { SWODE } & \text { BAB } \\ \text { HEALCH } & \text { SED } \\ \text { SAFT } & \text { SEMT } \\ \text { PAME } & \text { ZEB }\end{array}$

WET DRY

BACON EGGS

GREEN GRASS

DEEP

BOY

LORD

ARMY

RELIGION

EIGHT

LIGHT

EAST

MAXIMUM

STORY

MOUNTAIN

QUESTION

HERE

COFFEE

SWEET

GIVE

\section{POLT \\ POOB \\ CHESH \\ DEG \\ BAB \\ SED \\ SEMT}

DRY
EGGS
GRASS

SHALLOW

GIRL

LADY

NAVY

CHURCH

NINE

DARK

WEST

MINIMUM

BOOK

HILL.

ANSWER

WOULD

TAKE

BUTTER

TEA
Differing from word by more than one letter

\begin{tabular}{lr} 
FUNST & PROASH \\
CRINT & CHORDIN \\
PEDA & BUPON \\
THREG & IPSILV \\
RIMSARN & CHEPAL \\
TALFIN & PURP \\
THRIME & RULAT \\
THOLK & SHUSP \\
SAPUT & TEKAL \\
SEK & TUNST \\
& \\
NEGATIVE & \\
FINGERS & POSITIVE \\
FAT & TOES \\
CHILD & THIN \\
NEWS & BABY \\
APPLES & PAPER \\
HOUSE & PEARS \\
WAR & GARDEN \\
HOLLY & PEACE \\
FAST & BERRY \\
MOTHER & SLOW \\
SORROW & FATHER \\
TOP & JOY \\
COAT & BOTTOM \\
AIRCRAFT & HAT \\
CHEESE & PLANE \\
BREAD & BETA \\
ALPHA & WEB \\
BLACK & BISCUITS \\
& SOUR \\
\hline
\end{tabular}

(Manuscript received September 4, 1983;

revision accepted for publication April 16, 1984.) 